\title{
Seismogenic structure behaviour revealed by spatial clustering of seismicity in the Umbria-Marche Region (Central Italy)
}

\author{
Patrizia Tosi \\ Istituto Nazionale di Geofisica, Roma, Italy
}

\begin{abstract}
Time variations in the spatial distribution of earthquake epicentres are analyzed by application of the fractal correlation dimension method. The zone under investigation is located in Central Italy, bounded in longitude by 12.0 and 14.4 degrees east and in latitude by 42.0 and 43.6 degrees north. From 1st January 1978 to 5 th October 1997, 2028 events with a magnitude above $M_{l}=2.5$ constitute the database. Evolution of the spatial fractal dimension $D_{s}$ permits the identification of seismic cycles that are connected to the occurrence of main earthquakes. In particular, it is possible to recognize a division within each cycle, between a period of random background seismicity and a spatial clustering of events where shocks of magnitude $M_{l} \geq 4.0$ occur. Moreover, the decrease in $D_{s}$ prior to such events, evidences a structural relationship between foreshocks and the occurrence of a main shock, even if not in close territorial proximity. This feature indicates a new, more extensive definition of seismogenic structure which can includes several interconnected structures within a large area.
\end{abstract}

Key words seismogenic structure - fractal dimension - spatial clustering - foreshock - Central Italy

\section{Introduction}

Spatial distribution of seismicity has been investigated by several authors using a diverse range of techniques, from simple geographical representation to sophisticated analytical studies. The spatial localization of earthquakes constitutes the basis for a wide range of analysis, from source definition to seismotectonic considerations. Methods of examination involve a sequence of seismicity simplification,

Mailing address: Dr. Patrizia Tosi, Istituto Nazionale di Geofisica, Via di Vigna Murata 605, 00143 Roma, Italy; e-mail: tosi@ingrm.it such as to consider the seismic source as a point in space (this can be valid for the lower magnitude events), or to ignore the event depth when its accuracy is inferior to that of the geographic coordinates, or to assume that the effects of catalogue incompleteness or bias in localization introduced by the geometry of the seismic network are not critical. These specific limitations coexist with more general difficulties, where the study of a pattern of points involves arguments such as the definition of randomness, homogeneity, order, and clusterization. Even by way of a simple geographic inspection, the localization of seismic events has appeared to be non random in many cases, revealing a strict relationship to tectonic features such as faults, subduction slabs or volcanic systems. Where the tectonic structure can be identified by the location of individual hypocentres, it does not seem particularly important to further investigate the spatial distri- 
bution. Nevertheless, there are many situations in which spatial distribution seems to be homogeneous, or with an intermediate character between randomness and any specific structure. Whereas the random arrangement of a set of points can be considered homogeneous, a regular grid of points can also be considered homogeneous even though it is absolutely not random. These intrinsic difficulties led to the definition of several statistical parameters to define points distribution (Kagan and Knopoff, 1976; Prozorov and Dziewonski, 1982; Matsumura, 1984; Eneva et al., 1989, 1992; Tosi et al., 1994).

The last 20-30 years have seen a growing interest in the evidence of the fractal properties of seismicity, from energetic, temporal and spatial points of view (Smalley et al., 1987; Hirata and Imoto, 1991; Kagan and Jackson, 1991; Gabrielov and Newman, 1994). The fractal approach has introduced a sophisticated statistical tool to quantify the dimensional distribution of seismicity and with that, the properties of randomness and clusterization. In fact, these two terms can be set at the extremes of a continuous scale. Under the evolutionary aspect, the study of the temporal variations in the fractal dimension has also been useful to quantify the seismic process (Hirata, 1989a; Rossi, 1990; Radulian and Trifu, 1991; De Rubeis et al., 1993; Legrand et al., 1996). It is important to consider that a fundamental characteristic of a fractal is scale invariance (Mandelbrot, 1983). Therefore, the Gutenberg-Richter relation is also an expression of the self-similarity of seismic energy (Gabrielov and Newman, 1994; Chen et al., 1998). Under the spatial aspect, it has been demonstrated that faults are scale invariant with a fractal dimension peculiar to a specific zone (Okubo and Aki, 1987; Hirata, 1989b; Matsumoto et al., 1992). Time variations of the spatial fractal dimension of seismic events have been investigated, highlighting a connection with the $b$-value of the Gutenberg-Richter relation (Hirata, 1989a; Main, 1991; Lomnitz-Adler, 1992; Main, 1992; Öncel et al., 1996). This work investigates the spatial fractal dimension $\left(D_{s}\right)$ of epicentres, focusing on the time variation of this parameter in a zone of Central Italy where a sequence of several earthquakes recently struck the territory over a period of several months. The intention of this paper is to update and extend previous research done within the area (De Rubeis et al., 1993).

\section{Method}

The fractal dimension is a characteristic index of a fractal object. There are many definitions of fractal dimension, reflecting different aspects of the scale invariance and various methods to obtain it. The common element is the power-law relation between some aspect of the object and its measure. The generalized dimension $D_{q}$ of a fractal set is defined as

$$
D_{q}=\frac{1}{q-1} \lim _{r \rightarrow 0} \frac{\log \left(\sum_{i}\left(p_{i}(r)\right)^{q}\right)}{\log r}
$$

where $p_{i}(r)$ is the probability of a point belonging to the $i$ th box with size $r$. The real number $q$ is a weight that permits investigation of more or less densely clustered parts of the set. If $q=2, p_{i}$ represents the probability that 2 points are in the same box (Schuster, 1988) the corresponding $D_{2}$ is called the correlation dimension. Grassberger and Procaccia (1983) introduced the correlation integral to experimentally obtain the correlation dimension of a set of points $N$ as

$$
C(r)=\frac{2 N_{R<r}}{N(N-1)}
$$

where $N_{R<r}$ represents the number of point pairs separated by a distance $R$ less than $r$. If the distribution is fractal the following relation holds:

$$
C(r) \approx r^{D_{2}} .
$$

The algorithm used to evaluate $D_{2}$ calculates the slope of the best straight line fitting $C(r)$ versus $r$ on the log-log plot.

Theoretical fractals display a straight line in the $\log$-log plot for virtually any interval of 
$\log r$; for the experimental case there are lower and upper limits, due respectively to the minimum and maximum distance among pairs. Extreme care must be used in choosing the interval into which the slope has to be calculated: for the minimum distance values must not be lower than the local precision of the events. For the upper boundary, the most important constraint is the size and shape of the region investigated. There is a risk of under-sampling if values close to the maximum pair distance are set, due to the fact that the number of pairs is limited by the boundaries of the area. It is usually advised to avoid distances greater than $30 \sim 50 \%$ of the maximum distance found (Dongsheng et al., 1994). These considerations lead to a minimum and maximum distance allowed, but often a more severe constraint arises from a check of the $\log$-log plot of $C(r)$ versus $r$ : the lower and upper limit, into which the slope is to be calculated, must satisfy the definition of eq. (2.3), i.e. only the straight portion of the plot is valid for $D_{2}$ calculation.

Possible values of fractal dimension are bound to a range that is dependent on the dimension of the embedding space. Therefore, as only the geographical coordinates of the epicentres are under consideration in this instance, there is an upper limit of 2 and a lower limit of 0 . The interpretation of such limit values is that a set with $D_{2}=0$ has all events concentrated (clustered) into one point; at the other end of the scale, $D_{2}=2$ indicates that the events homogeneously cover the two-dimensional embedding space. As $D_{2}$ is calculated in the spatial domain, hereinafter it will be referred to as $D_{s}$.

The aim of this work is to investigate the time variation of $D_{s}$. There are two methods to achieve this, both based on the repeated evaluation of a moving window: one is to consider a fixed time window, the other is to consider a fixed number of events window. As an evaluation based on a fixed number of events prevents false oscillation of $D_{s}$, the latter method has been adopted here. After the calculation has been made inside a specific window, the corresponding fractal dimension value is assigned to the time of the last event under consideration.
A delicate parameter to be set is the number of points to be used inside every moving window. The best value should meet two opposite needs: a large number gives more reliable results, while a lower number of points gives more resolution in time. For this and other considerations expressed in De Rubeis et al. (1993, and references therein), a window of 40 events was chosen for the analysis.

\section{Data}

The earthquakes investigated belong to an area of Central Italy bounded in longitude by 12.0 and 14.4 degrees east and in latitude by 42.0 and 43.6 degrees north. The events pertain to a time period spanning from 1st January 1978 to 5th October 1997, as extracted from the Italian National Catalogue, recorded by the permanent seismic network run by the Istituto Nazionale di Geofisica. Magnitude comprises values above $M_{l}=2.5$ (when $M_{l}$ was not available $M_{d}$ was applied): a total of 2028 events constitutes the current database. For this Apennines area, the event depth is generally within $30 \mathrm{~km}$ and the epicentre localization error is less than $10 \mathrm{~km}$. Completeness of the database has been verified using the method introduced by Mulargia et al. (1987). Figure 1 shows localization of the epicentres: event locations reveal a pattern of structures principally aligned in a NW-SE trend (this direction is coincident with the Apennine mountain chain), moreover clusters are present and a minor number of quakes appear to be randomly placed.

The tectonics of the region, as for most of the Apennine chain, are affected by the Quaternary activity of normal faults oriented NW-SE. The fault-plane solutions of the focal mechanism are in agreement, indicating for the major events in this zone, normal faults with the same direction and dipping to the southwest (Deschamps et al., 1984; Ekström et al., 1998). There is also evidence of a NE-SW transversal fault system, both extensional and strike-slip.

A fractal analysis of all earthquakes was performed. The aim was to check if the whole data set displayed a fractal character and the 


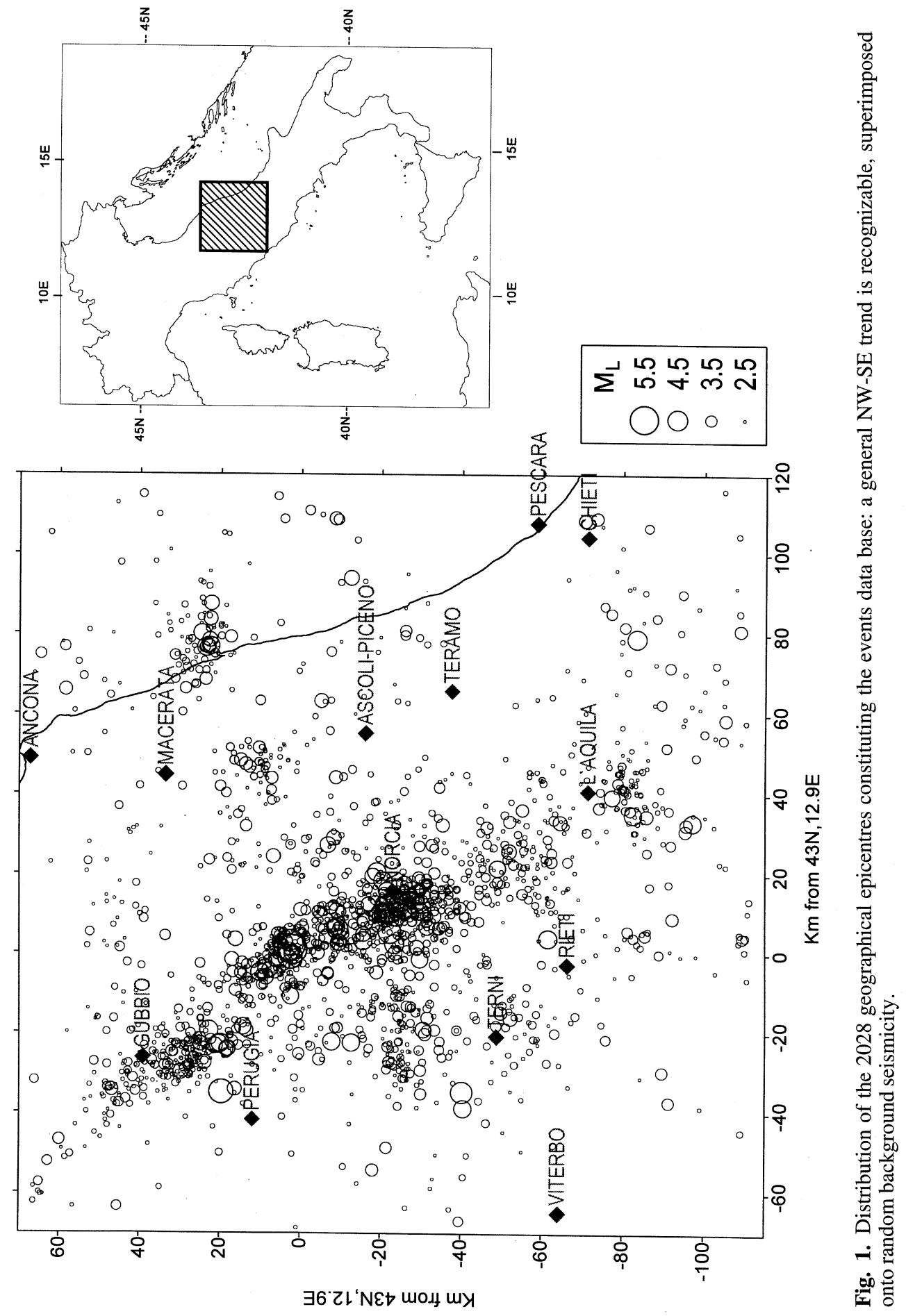




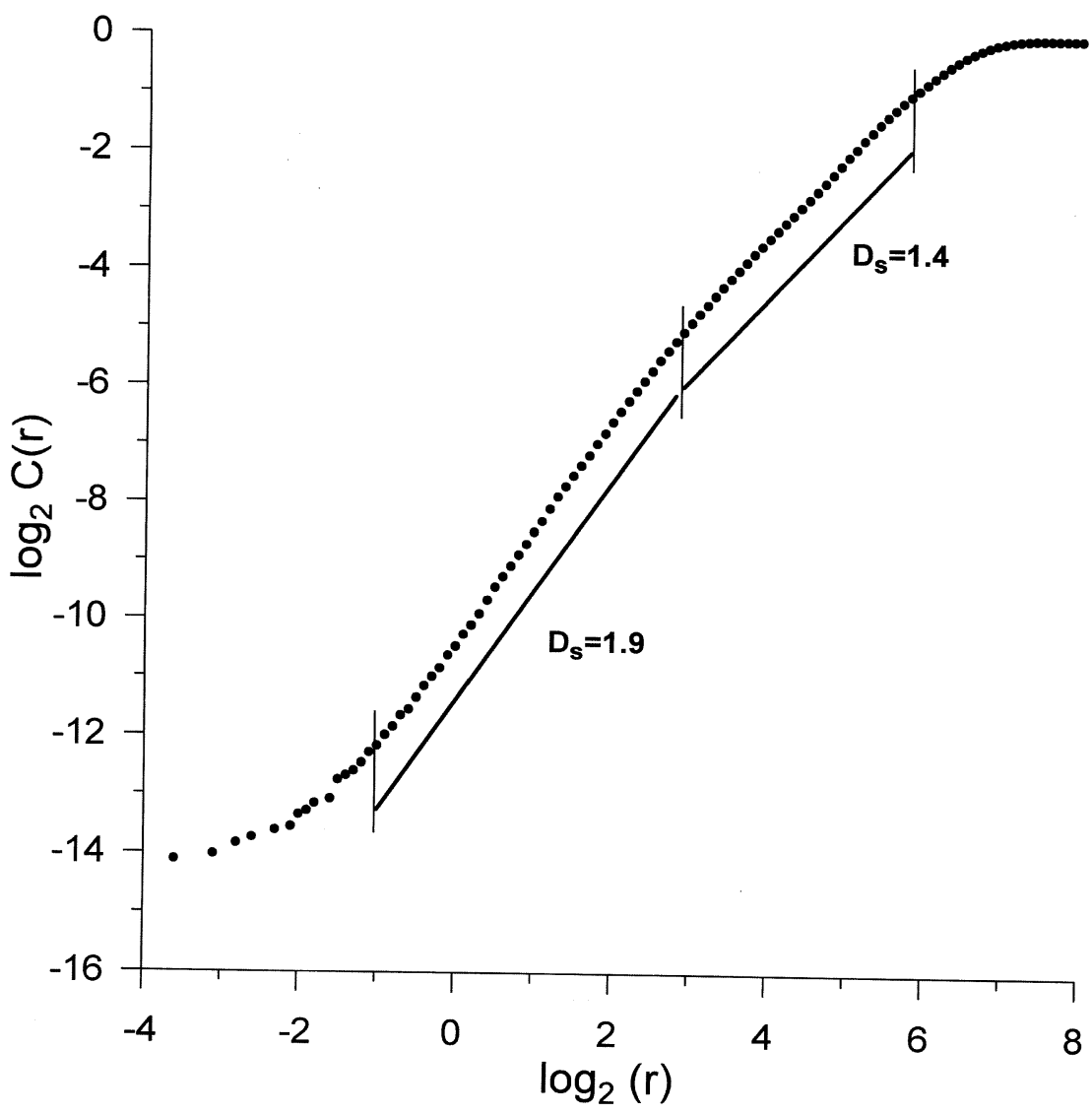

Fig. 2. Log-log plot of $C(r)$ versus $r$ (in kilometers) for the whole data set. Two linear sections are distinguishable at different spatial ranges: the shortest range of 0.5-7 km falls within the zone of error in event location and indicates a fractal dimension equal to 1.9; the second range of 8-56 km indicates a more clustered setting $\left(D_{s}=1.4\right)$.

relative bounds of the spatial range. The relative $\log$ - $\log$ plot of $C(r)$ versus $r$ is depicted in fig. 2. It is possible to recognize two distinct straight portions: the first in the range 0.5$7 \mathrm{~km}$, the second in the range 8-56 km, with fractal dimensions of $D_{s}=1.9$ and $D_{s}=1.4$ respectively. The first range identifies a region completely inside the estimated error of events location and this is supported by our analysis. In fact, the corresponding fractal dimension is near to the value of the embedding space and is quite high, due to the superimposition of random error (by definition with high $D_{s}$ ) on the true localization. The second range extends from the end of the random range, to an upper boundary value of about $56 \mathrm{~km}$. This upper limit is probably due to border effects, as after this value the log-log plot clearly shows a saturation effect. In other words, the numerator of the expression (2.2) does not increase, as happened for lesser distances, denoting a lack of point pairs above this range (note that this response continues until the $130 \mathrm{~km}$ value is reached, above which no more pairs exist). The fractal dimension of this second range is 1.4, indicating a certain degree of clusterization and the presence of non random structures. 


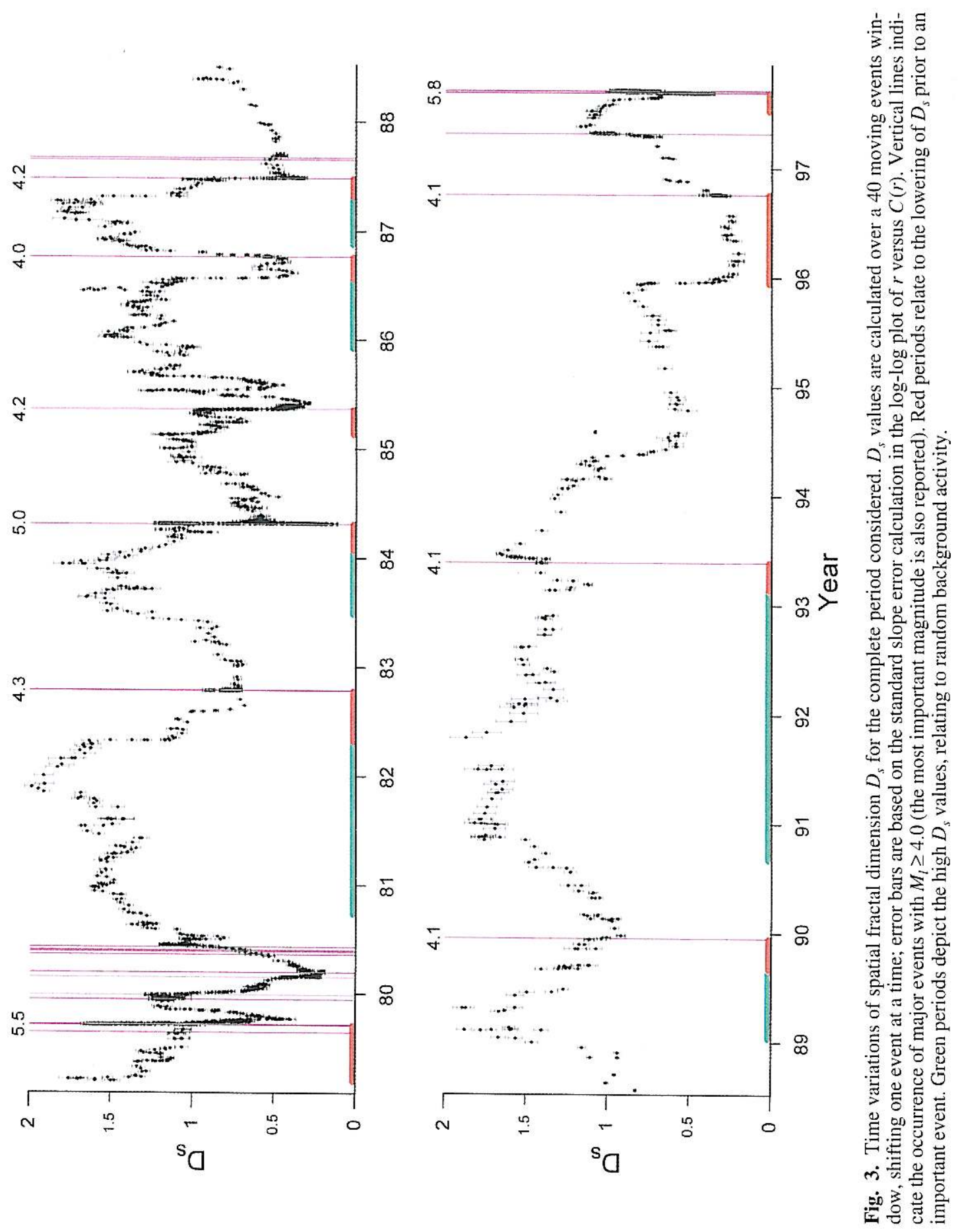




\section{Results}

An investigation of the time variations of $D_{s}$ was performed using overlapping time windows of 40 events. The shift between successive windows was set at 1 event, permitting a sufficient smoothing among $D_{s}$ values. $D_{s}$ was calculated in the spatial range $16-45 \mathrm{~km}$.

Figure 3 shows the variations of $D_{s}$ over the complete period analyzed. In general, values span almost the entire permitted range of variability (0-2), showing variations in several time scales. In abscissa there is time in years and, superimposed on the plot, there is the time occurrence of the events of magnitude $M_{l} \geq 4.0$ (1.5 higher the minimum magnitude considered).

Global behaviour follows this pattern: due to the clustered sequence that follows a main shock, a local minimum of $D_{s}$ (usually $D_{s} \leq 1$ ) marks a short period of time immediately following the occurrence of an earthquake with a magnitude of 4.0, or greater. After this phase, the $D_{s}$ increases, reaching a local high $\left(D_{s}>1\right)$.
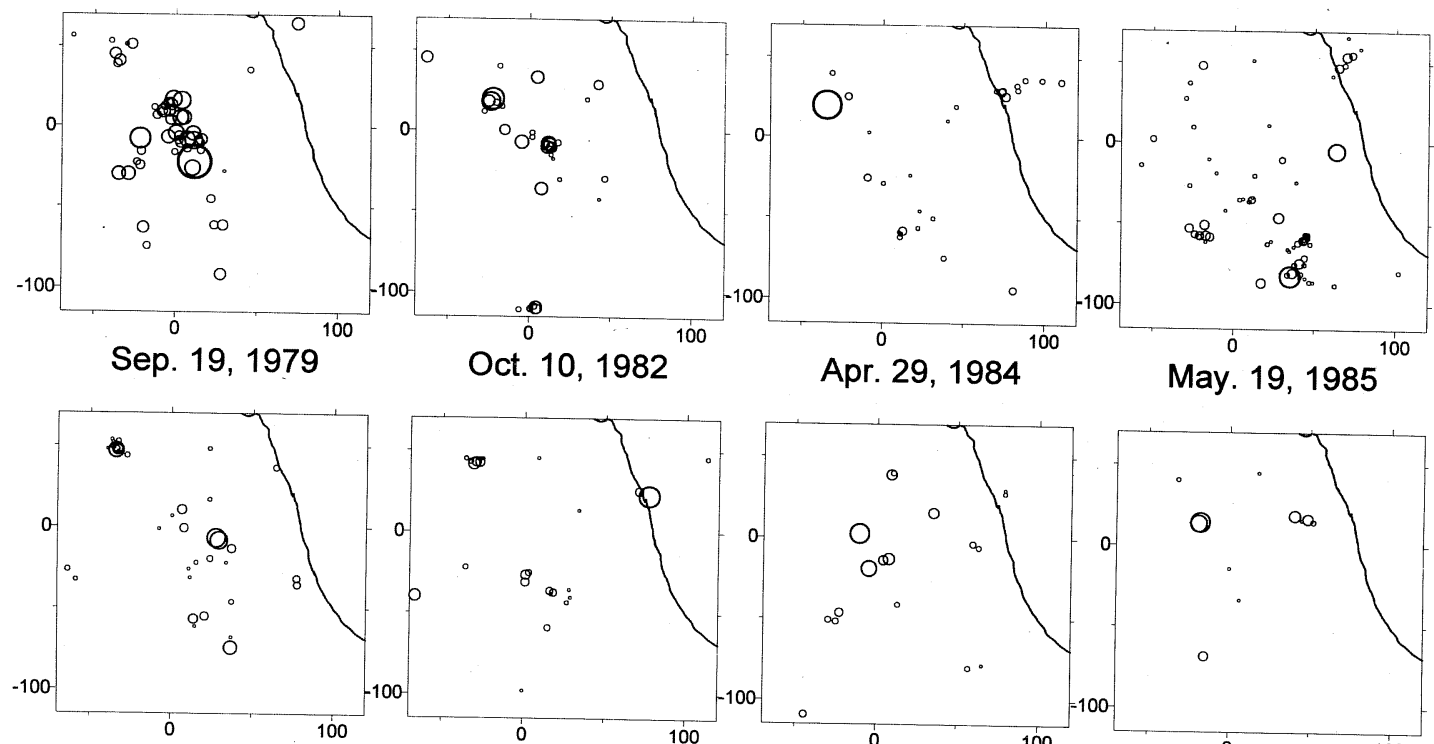

Oct. 13,1986

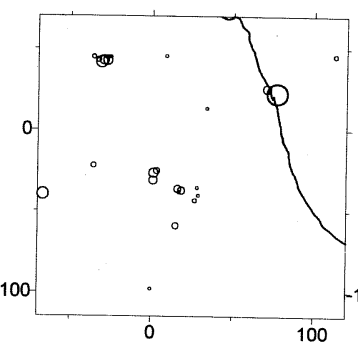

Jul. 3, 1987

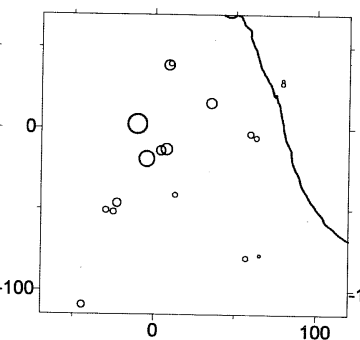

Dec. 12, 1989
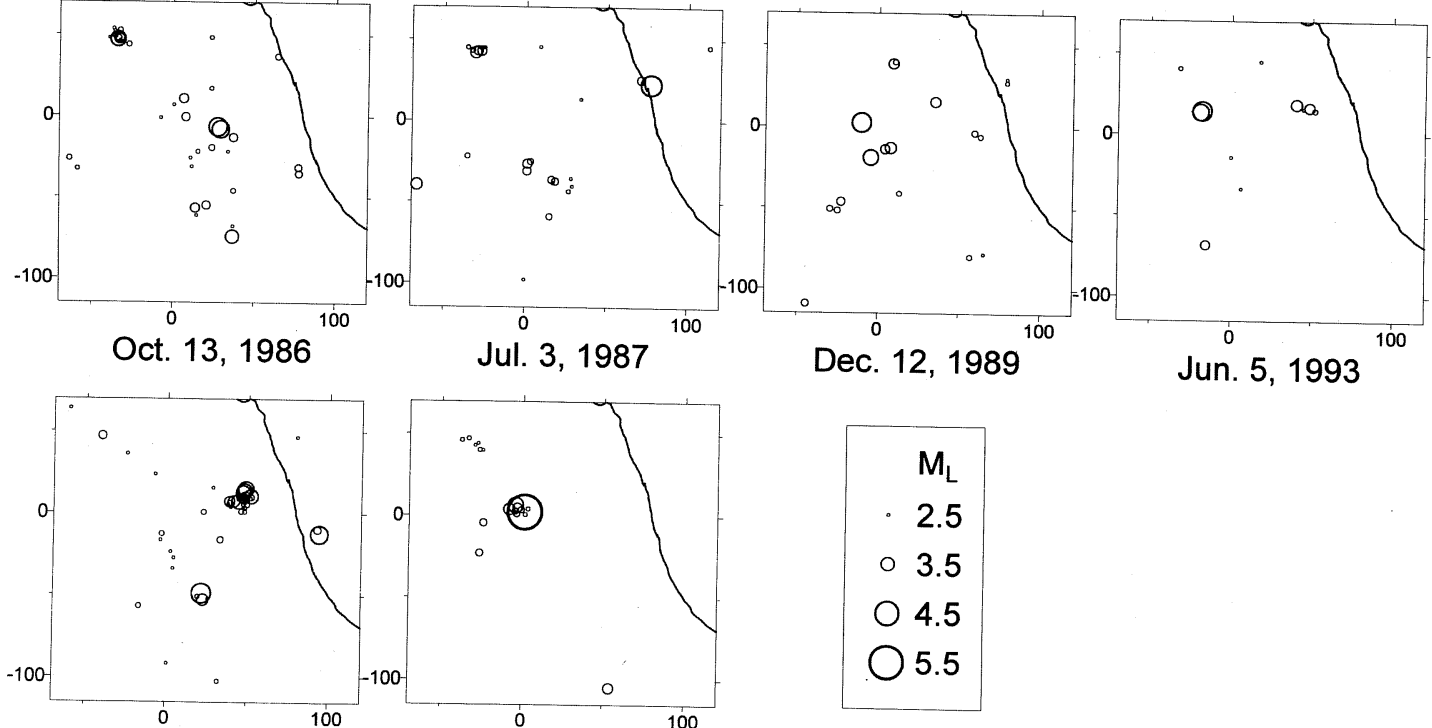

Oct. 20, 1996
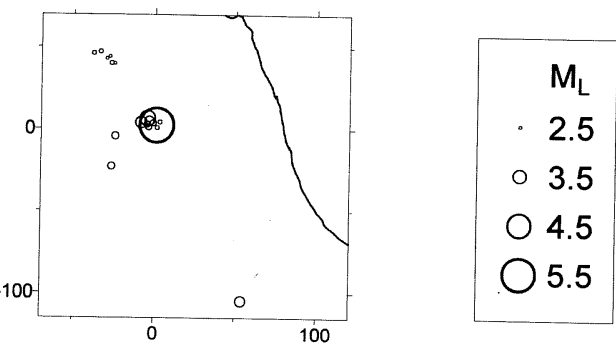

Jun. 5, 1993

Sep. 26, 1997

Fig. 4. Spatial distribution of the epicentres relative to the lowering $D_{s}$ values prior to an important seismic event (red periods in fig. 3). In each plot the biggest circle marks the location of the main event which occurred at the end of the interval considered. In general, distinct clusters are activated prior to the main shock.
Axes units are in kilometers. 
Upon reaching the maximum values, the $D_{s}$ tends to decrease, until a new event occurs and the cycle repeats itself.

While it is not surprising that the low $D_{s}$ values are related to the evolution of the seismic sequence activated by a main shock, it is interesting to further analyze the other $D_{s}$ behaviours. Towards the end of a sequence, a constant rise in $D_{s}$ is observed, suggesting that such a specific structure will lower the rate of clustered seismicity in favour of random background activity. In fact, high $D_{s}$ values are typical of random points distribution and do not appear to be related to a particular process. The highest $D_{s}$ values $(\approx 2)$ pertain specifically to background activity, which is not supposed to be associated to any particular structure.

The lowering of $D_{s}$ prior the occurrence of a strong event $\left(M_{l} \geq 4.0\right)$, is very interesting. It is inferred that the earthquakes causing the lowering seem to be associated to the main event and can therefore be considered foreshocks. A detailed analysis of this phase of the evolution is shown in fig. 4, where the epicentre locations of the preceding earthquakes which have caused a lowering of $D_{s}$ values, were plotted for every strong event. A series of clustered epicentres, not necessarily located at a short distance from the main shock, are remarkably evident. This is an important result as it gives new insights into the definition of a seismic structure, not constrained by distance. For example, Console et al. (1993), give the spatial limit of a $30 \mathrm{~km}$ radius to recognize a foreshock, Jones (1985) and later Agnew and Jones (1991) found a $10 \mathrm{~km}$ range within which a significant percentage of foreshocks occur. Our analysis shows a structure activation of 2-3 months duration, even longer in some cases, before an event of $M_{l} \geq 4.0$. The important result is that in revealing foreshock activity at distances greater than $100 \mathrm{~km}$, the structure is not strictly limited to the vicinity of the main shock. All the events preceding main shocks are plotted in fig. 5a, in comparison, the events pertaining to high values of $D_{s}$ are shown in fig. $5 \mathrm{~b}$. The differences between the two groups of seismic activity are evident with several well defined clusters in fig. 5a, while in fig. 5b seismicity appear to be homogeneously distributed and does not reveal any link with seismogenic structures.
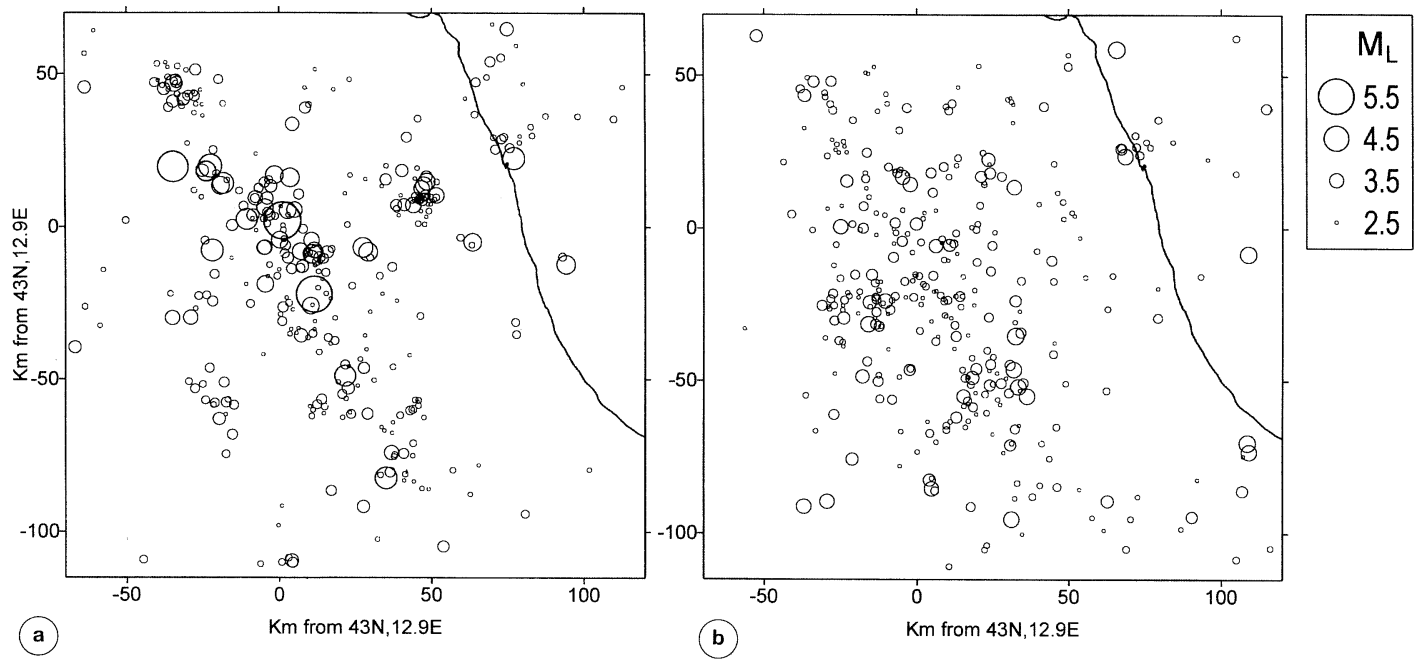

Fig. 5a,b. a) Represents the whole set of events depicted in fig. 4 (red periods in fig. 3); b) depicts the whole set of background activity (green periods of fig. 3). Differences in the spatial localization between the two groups (clustering in (a) and homogeneity in (b)) are evident. 


\section{Conclusions}

This paper constitutes the further development of a previous analysis (De Rubeis et al., 1993). The patterns in the spatial behaviour are confirmed for the earlier events of magnitude $4.0 \leq M_{l}<4.5$ and also for the more recent seismicity. Analysis of the decreasing of $D_{s}$ prior to the occurrence of high energy events has been extended to emphasize the presence of links within seismogenetic structures. Data and results show evidence of a complex seismotectonic structure behaviour: in particular the spatial influence of a main shock does not appear to be strictly confined to the area surrounding the event. Activity concentrated around the epicentre of a strong event is certainly present during the aftershock sequence and is the local answer to the stress/strain disequilibrium. Concerning the period of time preceding an important event, seismicity appears to act as a non random process, evidencing the recurrent clusterization of events over a wide area in locations that are relatively common. This is probably due to the activation of several interconnected seismogenic structures, within an area of circa $90 \mathrm{~km}$ in radius.

The spatial fractal dimension is shown to be an efficient statistical parameter to reveal seismic spatial behaviour. Application of this parameter allows random background activity, aftershock sequence and foreshocks, to be readily distinguished and characterized. The spatial ranges where the whole set of seismicity is fractal, permits a clear distinction between two phases: a short range with high $D_{s}$ values, reflecting the uncertain location of the epicentre and an intermediate spatial range into which the events as a whole appear not to be randomly localized. More extensive spatial ranges cannot be investigated due to the limiting border effects of the examined area.

Main (1991, and references therein) has shown that the fractal distribution of earthquakes can be a consequence of a self organized critical process: this requires the presence of a long range interaction in the fracture process (Rundle and Klein, 1989), or a long range order produced by short range interactions (Bak and Tang, 1989). The results of this paper indicate that the whole seismic process, being the critical response to a stress field, is a large area process; possible precursors must therefore be investigated within the whole structure, without short range restrictions.

\section{Acknowledgements}

I am grateful to V. De Rubeis for the constructive discussion of the results of this paper. I thank P. Dimitriu for helpful suggestions and S. Watt for the English revision.

\section{REFERENCES}

AGNEW, D.C. and L.M. JoNES (1991): Prediction probabilities from foreshocks, J. Geophys. Res., 96, 11959. 11971.

BAK, P. and C. TANG (1989): Earthquakes as a self-organized critical phenomenon, J. Geophys. Res., 94, 15635-15637.

Chen, Y., L. Chen, Z. LiU and R. Wu (1998): A new fractal approach to the clustering of earthquakes: physical fractal, Bull. Seism. Soc. Am., 88, 89-94.

Console, R., M. Murru and B. Alessandrini (1993): Foreshocks statistics and their possible relationship to earthquake prediction in the Italian region, Bull. Seism. Soc. Am., 83, 1248-1263.

De Rubeis, V., P. Dimitriu, E. Papadimitriou and P. TOSI (1993): Recurrent patterns in the spatial behavior of Italian seismicity revealed by the fractal approach, Geophys. Res. Lett., 20, 1911-1914.

DESCHAMPS, A., G. IANNACCONE and R. SCARPA (1984): The Umbrian earthquake (Italy) of 19 September 1979, Ann. Geophysicae, 2, 29-36.

DONGSHENG, L., Z. ZHAOBI and W. BinghONG (1994): Research into the multifractal of earthquake spatial distribution, Tectonophysics, 233, 91-97.

Ekström, G., A. Morelli, E. Boschi and A. DzIEwonsKi (1998): Moment tensor analysis of the Central Italy earthquake sequence of September-October 1997, Geophys. Res. Lett., 25, 1971-1974.

ENEVA, M. and M.W. HAMBURGER (1989): Spatial and temporal patterns of earthquake distribution in Soviet Central Asia: application of pair analysis statistics, Bull. Seism. Soc. Am., 79, 1457-1476.

Eneva, M., M.W. HAMBurger and G.A. Popandopulo (1992): Spatial distribution of earthquakes in aftershock zones of the Garm region, Soviet Central Asia, Geophys. J. Int., 109, 38-53.

GABRIELOV, A. and W.I. NEWMAN (1994): Seismicity modeling and earthquake prediction: a review, in Nonlinear Dynamics and Predictability of Geophys. Phenom., Geophys. Monogr. 83, IUGG, 18, 7-13.

Grassberger, P. and I. ProcacCIA (1983): Measuring the strangeness of strange attractors, Physica D, 9, 189-208. 
HiRATA, T. (1989a): A correlation between the $b$ value and the fractal dimension of earthquakes, J. Geophys. Res., 94, 7507-7514.

HiRATA, T. (1989b): Fractal dimension of fault systems in Japan: fractal structure in rock fracture geometry at various scales, Pageoph, 131, 157-170.

Hirata, T. and M. IMOTo (1991): Multifractal analysis of spatial distribution of microearthquakes in the Kanto region, Geophys. J. Int., 107, 155-162.

JONES, L.M. (1985): Foreshocks and time-dependent earthquake hazard assessment in Southern California, Bull. Seism. Soc. Am., 75, 1669-1680.

KAGAN, Y.Y. and D.D. JACKSON (1991): Long-term earthquake clustering, Geophys. J. Int., 104, 117-133.

KAGAN, Y.Y. and L. KNOPOFF (1976): Statistical search for non-random features of the seismicity of strong earthquakes, Phys. Earth Planet. Int., 12, 291-318.

Legrand, D., A. Cisternas and L. Dorbath (1996): Multifractal analysis of the 1992 Erzincan aftershock sequence, Geophy. Res. Lett., 23, 933-936.

LOMNITZ-ADLER, J. (1992): Interplay of fault dynamics and fractal dimension in determining Gutenberg \& Richter's b-value, Geophys. J. Int., 108, 941-944.

MAIN, I.G. (1991): Damage mechanics with long-range interactions: correlation between the seismic $b$-value and the fractal two-point correlation dimension, Geophys. $J$. Int., 107, 531-541.

MAIN, I.G. (1992): A modified Griffith criterion for the evolution of damage with a fractal distribution of crack lengths: application to seismic event rates and $b$-values, Geophys. J. Int., 111, 353-362.

MANDELBROT, B.B. (1983): The Fractal Geometry of Nature (W.H. Freeman, New York), 109-115.

Matsumoto, N., K. Yomogida and S. HondA (1992): Fractal analysis of fault systems in Japan and the Philippines, Geophys. Res. Lett., 19, 357-360.

MATSUMURA, S. (1984): A one-parameter expression of seismicity patterns in space and time, Bull. Seism. Soc. Am., 74, 2559-2576.

Mulargia, F., P. GASPERINI and S. TinTI (1987): Contour mapping of Italian seismicity, Tectonophysics, 142, 203-216.

OKUBO, P.G. and K. AKI (1987): Fractal geometry in the San Andreas fault system, J. Geophy. Res., 92, 345-355.

ÖNCEL, A.O., I. MAIN, Ö. AlPTEKIN and P. COWIE (1996): Temporal variations in the fractal properties of seismicity in the North Anatolian fault zone between $31^{\circ} \mathrm{E}$ and $41^{\circ} \mathrm{E}$, Pageoph, 147, 147-159.

Prozorov, A.G. and A.M. DzIEwONSKI (1982): A method of studying variations in the clustering property of earthquakes: application to the analysis of global seismicity, J. Geophys. Res., 87, 2829-2839.

Radulian, M. and C. TRIFU (1991): Would it have been possible to predict the 30 August 1986 Vrancea earthquake?, Bull. Seism. Soc. Am., 81, 2498-2503.

RossI, G. (1990): Fractal dimension time variations in the Friuli (Northeastern Italy) seismic area, Boll. Geofis. Teor. Appl., 32, 175-183.

RuNDLE, J.B. and W. KLEIN (1989): Nonclassical nucleation and growth of cohesive tensile cracks, Phys. Rev. Lett., 63, 171-174.

SCHUSTER, H.G. (1988): Deterministic Chaos (VCH Verlagsgesellschaft), 117-131.

Smalley, R.F., J.L. Chatelain, D.L. Turcotte and R. PREVOT (1987): A fractal approach to the clustering of earthquakes: applications to the seismicity of the New Hebrides, Bull. Seism. Soc. Am., 77, 1368-1381.

Tosi, P., V. De Rubeis, E. PAPAdimitriou and P. DimiTRIU (1994): Statistical study of epicentre alignment in the broader Aegean area, Ann. Geofis., 37 (5), 939-948.

(received April 3, 1998;

accepted June 22, 1998) 